\title{
Folding Single Chains to Single-Chain Nanoparticles via Reversible Interactions: What Size Reduction Can One Expect?
}

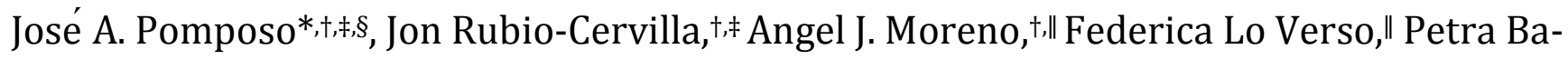 \\ cova, ${ }^{\dagger}$ Arantxa Arbe, ${ }^{\dagger}$ and Juan Colmenero ${ }^{\dagger, \ddagger}, \|$ \\ ${ }^{\dagger}$ Centro de Física de Materiales (CSIC, UPV/EHU) and Materials Physics Center MPC, Paseo Manuel de Lardizabal 5, E- \\ 20018 San Sebastián, Spain \\ ‡ Departamento de Física de Materiales, Universidad del País Vasco (UPV/EHU), Apartado 1072, E-20800 San Sebas- \\ tián, Spain \\ § IKERBASQUE - Basque Foundation for Science, María Díaz de Haro 3, E-48013 Bilbao, Spain \\ "Donostia International Physics Center (DIPC), Paseo Manuel de Lardizabal 4, E-20018 San Sebastián, Spain \\ Supporting Information
}

\begin{abstract}
Single-chain nanoparticles (SCNPs) constructed via reversible bonds are versatile stimuli-responsive soft nanoobjects with potential use in nanomedicine, bioimaging, biosensing and catalysis applications. In recent years, many different types of reversible SCNPs have been reported involving intra-chain hydrogen bonding, host-guest interactions and metal complex formation, among other reversible bonds. As illustrated in this work, reversible SCNPs in solution with similar nature, molar mass and amount of reactive groups than irreversible (covalentbonded) SCNPs display, on average, a lower level of chain compaction. We follow herein a Flory-like argument to obtain a simple expression providing the expected

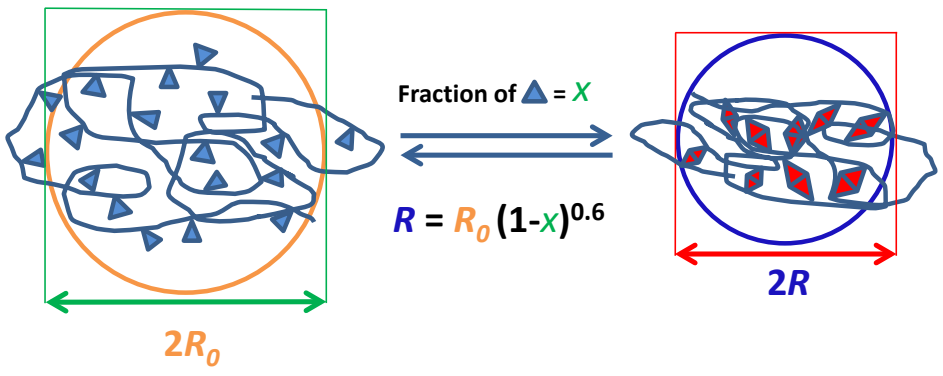
size reduction upon folding single chains of size $R_{0}$ to SCNPs of size $R$ with reversible interactions. Accurate estimation of $R$ is of outmost importance for developing practical applications of responsive SCNPs based on structure - property relationships. For precursor chains having a fraction of groups $x$ involved in reversible bonds, the expected size upon intra-chain folding of the precursor chains to SCNPs is given by $R=R_{0}(1-x)^{0.6}$. We perform a comparison of the size reduction predicted by the former expression with extensive literature data for SCNPs constructed via reversible bonds (65 SCNPs, 16 reversible interactions). The overall agreement between theoretical and experimental data is excellent, hence allowing a valuable a priori estimation of the size reduction upon folding single chains to single-chain nanoparticles via reversible interactions.
\end{abstract}

\section{INTRODUCTION}

Single-chain nanoparticles (SCNPs) are the result of applying single-chain technology to individual polymer chains, giving rise to versatile folded/collapsed soft nano-objects of ultra-small size (3-20 nm). ${ }^{1-12}$ Significant effort has been spent in last years to endow SCNPs with useful and bioinspired applications for the fields of nanomedicine, ${ }^{13,14}$ bioimaging, ${ }^{15,16}$ biosensing, ${ }^{17,18}$ protein mimicry ${ }^{12,19-21}$ and catalysis, ${ }^{22-25}$ among others. In recent years, in addition to the preparation of permanent, covalentbonded SCNPs an increasing interest in the development of stimuli-responsive SCNPs constructed via reversible interactions has been generated. ${ }^{2-9,11}$

The feasibility to produce non-covalent bonded SCNPs via hydrogen bonding interactions was established in the pioneering work by Hawker, Kim and co-workers. ${ }^{26}$ Significant advances in the field were carried out by Palmans, Meijer et al. by utilizing ureido-pyrimidinone (UPy) dimerization ${ }^{27-30}$ and benzene-1,3,5tricarboxamide (BTA) helical stacking ${ }^{22,31-37}$ as intra-chain reversible interactions. Recently, intramolecular quadruple hydrogen bonding interactions have been employed by $\mathrm{Pu}$ et al. ${ }^{38}$ to obtain reversible SCNPs. Host-guest interactions involving a cucurbit[8]uril cage compound were exploited by Scherman and coworkers $^{39}$ to produce responsive SCNPs based on ternary complex formation between cucurbit[8]uril (host) and viologen and naphtyl moieties (guests). More recently, voltage-responsive SCNPs have been prepared by $\mathrm{Pu}$ et al. ${ }^{40}$ via cyclodextrinferrocene host-guest interactions. Concerning reversible covalent bonds, dynamic covalent SCNPs were synthesized by Murray and Fulton $^{41}$ in a pioneering work utilizing hydrazone reversible bonds. SCNPs prepared via reversible disulfide bridges were reported by Berda and coworkers. ${ }^{42}$ Very recently, redoxresponsive SCNPs have been constructed by Thayumanavan et $a l .{ }^{43}$ via intra-chain disulfide exchange reactions. $\mathrm{pH}$-responsive 
SCNPs were synthesized by Fulton, Pomposo and co-workers ${ }^{44}$ by exploiting dynamic covalent enamine bonds. More recently, anthracene dimerization has been introduced by Berda et al. ${ }^{45}$ for the construction of UV-light responsive SCNPs. Concerning metal complexation, several strategies have been developed to synthesize $\mathrm{Ru}-{ }^{22} \mathrm{Rh}-,{ }^{46} \mathrm{Cu}-,{ }^{47-50} \mathrm{Pd}-,{ }^{51} \mathrm{Ir}^{-} / \mathrm{Ni}_{-}{ }^{52}$ and $\mathrm{Fe}-$ ${ }^{53}$ containing SCNPs. All the above reversible SCNPs can be disassembled by means of one or several appropriate stimuli (e.g., temperature increase, addition of competitive host compounds, $\mathrm{pH}$ decrease/increase, redox potential changes).

For covalent-bonded (irreversible) SCNPs, a detailed analysis of the size reduction upon SCNP formation was previously carried out. $^{54}$ The analysis revealed that, in general, permanent SCNPs in solution adopt open, sparse morphologies resembling those typical of intrinsically disordered proteins, instead of compact globule morphologies. ${ }^{54} \mathrm{~A}$ similar in-depth analysis of size reduction for SCNPs constructed via reversible bonds has not yet been performed. In this work, we perform such an analysis and use a Flory-like argument to derive a simple expression providing the expected size reduction upon folding single chains of size $R_{0}$ to SCNPs of size $R$ via reversible interactions. Subsequently, we perform a comparison of the predicted size reduction to extensive experimental data taken from the literature concerning SCNPs constructed via reversible bonds. Also, a comparison of the level of chain compaction between reversible and irreversible SCNPs of the same nature, molar mass and amount of reactive groups is performed.

\section{RESULTS AND DISCUSSION}

Table 1 summarizes the relevant data for the different 65 responsive SCNPs (16 different types of reversible interactions) that we are considering in this work. As it is described in the corresponding references and compiled in the Supporting Information, SI (Table S1), all these SCNPs were synthesized under the following conditions: (i) good solvent conditions; (ii) high dilution $(\leq 1$ $\mathrm{mg} / \mathrm{mL}$ ) far below the overlap concentration $\mathrm{c}^{*}$, where $\mathrm{c}^{*}$ is typically in the range $30-100 \mathrm{mg} / \mathrm{mL}$; (iii) reversible crosslinking interactions. Under these conditions, the formation of intermolecular aggregates is statistically very infrequent because there is not driving force to form them. Unlike in the case of bad solvent conditions, in terms of energy there will be no preference for intermolecular aggregation that will compensate the associated entropic loss. Anyway, the single-chain nature of these nanoparticles was checked by means of different experimental techniques (see the corresponding references) as it is summarized in Table S1 of the SI.

Table 1 includes the hydrodynamic size for the 65 SCNPs considered. The hydrodynamic size of the precursor $\left(R_{\mathrm{H}}^{\mathrm{SEC}}\right)$ was calculated according to: $R_{\mathrm{H}}{ }^{\mathrm{SEC}}(\mathrm{nm})=1.44 \times 10^{-2} M_{\mathrm{w}}^{0.561}$, which is the recommended expression for estimating the hydrodynamic radius based on $M_{\mathrm{w}}$ data from size exclusion chromatography (SEC) in tetrahydrofuran (THF) calibrated with PS standards. ${ }^{55}$ From the reported apparent weight average molecular weight ${ }^{56}$ from SEC measurements in THF $\left(M_{\mathrm{w}}{ }^{a p p}\right)$, the hydrodynamic radius of the SCNP was obtained as $R_{\mathrm{H}}^{\text {SEC }}(\mathrm{nm})=1.44 \times 10^{-2}\left(M_{\mathrm{w}}{ }^{a p p}\right)^{0.561}$. Hydrodynamic size data as determined from dynamic light scattering (DLS) measure- ments in THF $\left(R_{\mathrm{H}}{ }^{\mathrm{DLS}}\right)$ are also provided in Table 1 , when available.

To derive an expression providing the expected size reduction upon folding single chains of size $R_{0}$ to SCNPs of size $R$ via reversible interactions we follow a Flory-like argument. ${ }^{57}$ We consider a SCNP in good solvent with a number of segments $N$ and segment length $b$ having a fraction of groups $x$ involved in reversible bonds (Figure 1a). We obtain the free energy of this system, $F_{\mathrm{T}}$, by assuming that actually only the non-bonded segments, $(1-x) N$, contribute effectively to the excluded volume, $F_{\text {exc }}$, and entropic, $F_{\text {ent }}$, terms. Therefore, the Flory expression for the free energy ${ }^{57}$ is modified as:

$$
\begin{gathered}
F_{T} /\left(k_{B} T\right)=F_{\text {exc }} /\left(k_{B} T\right)+F_{\text {ent }} /\left(k_{B} T\right) \approx \\
\approx v(1-x)^{2} N^{2} / R^{3}+R^{2} /\left((1-x) N b^{2}\right)
\end{gathered}
$$

with $v$ the excluded volume parameter. By minimizing $F_{\mathrm{T}}$ with respect to $R$ we obtain:

$$
R=(3 / 2)^{1 / 5} v^{1 / 5} b^{2 / 5}(1-x)^{3 / 5} N^{3 / 5}
$$

$R_{0}$ results by putting $x=0$ in eq. 2 :

$$
R_{0}=(3 / 2)^{1 / 5} v^{1 / 5} b^{2 / 5} N^{3 / 5}
$$

Finally, from eq. 2 and 3 we have:

$$
R=R_{0}(1-x)^{0.6}
$$

Implicit in Eq. 4 is the approximation that, concomitant to SCNP formation, all potential intra-chain reversible bonds are formed. This is a good approximation, since the fraction of formed bonds should be about $\left[1-\exp \left(-\Delta E / \mathrm{k}_{\mathrm{B}} T\right)\right]$, and the energy scale $\Delta E$ of the reversible bonds is several tenths the thermal energy $\mathrm{k}_{\mathrm{B}} T$. Moreover, the assumption of an identical scaling exponent $v=0.6$ for SCNPs (Eq. 2) and precursors (Eq. 3) is valid only if chain compaction takes place mostly at the local scale and the global conformation remains selfavoiding. Eq. 4 is expected to suffer from the same type of shortcomings and error cancellations as in Flory's original theory. ${ }^{57}$ In spite of these limitations, we will show that this simple approach is in good agreement with literature results, providing a useful tool for predicting the size of SCNPs with reversible bonds.

The expected hydrodynamic size reduction according to eq. 4 is reported in Table 1 for different SCNPs involving reversible interactions. Inspection of data from Table 1 reveals that, in general, the agreement between hydrodynamic radii calculated from eq. $4\left(R_{\mathrm{H}}{ }^{\mathrm{CAL}}\right)$ and experimental hydrodynamic radii $\left(R_{\mathrm{H}}{ }^{\mathrm{EX}}\right)$ is very good. As a representative example, Figure 1b shows a plot of $R_{\mathrm{H}}{ }^{\text {CAL }}$ versus $R_{\mathrm{H}}^{\text {EXP }}$ for reversible polystyrene (PS)-SCNPs prepared via (i) ureido-pyrimidinone (UPy) dimerization, ${ }^{30}$ (Table 1 , entries 11,12 ) (ii) hydrazone reversible bonds, ${ }^{41}$ (Table 1 , entries 29-37) (iii) Cu-phthalocyanine formation $^{50}$ (Table 1, entries 54-56) and (iv) Pd- complexation $^{51}$ (Table 1, entry 57). Even considering SCNPs constructed from very different reversible bonds, the average deviation of calculated data from experimental ones is only $7 \%$. Addi- 
Table 1. Size Data for Different Single-Chain Nanoparticles (SCNPs) Constructed via Reversible Interactions Compared to Calculated Size Data from Eq. 4 (see Text)

\begin{tabular}{|c|c|c|c|c|c|c|c|c|c|c|c|c|c|}
\hline \multicolumn{7}{|c|}{ Precursors } & \multicolumn{7}{|c|}{ SCNPs } \\
\hline$\#$ & Type $^{a}$ & $x^{b}$ & $\begin{array}{c}M_{w} \\
(\mathbf{k D a}) \\
\mathbf{c}\end{array}$ & $\nexists^{d}$ & $\begin{array}{l}R_{H}{ }^{\text {SEC }} \\
(\mathbf{n m})^{\mathrm{e}}\end{array}$ & $\begin{array}{c}R_{H}^{\text {DLS }} \\
(\mathbf{n m})^{\text {f }}\end{array}$ & $\begin{array}{l}\text { Reversible } \\
\text { Interaction }^{\text {g }}\end{array}$ & $\begin{array}{c}\text { SCNP } \\
M_{w}{ }^{a p p} \\
(\mathbf{k D a})^{\mathrm{h}}\end{array}$ & $\begin{array}{l}R_{H}^{\text {SEC }} \\
(\mathbf{n m})^{\mathrm{e}}\end{array}$ & $\begin{array}{l}R_{H}{ }^{C A L} \\
(\mathrm{SEC}) \\
(\mathrm{nm})^{\mathrm{i}}\end{array}$ & $\begin{array}{l}R_{H}^{\text {DLS }} \\
(\mathbf{n m})^{\mathrm{f}}\end{array}$ & $\begin{array}{l}R_{H}^{\text {CAL }} \\
(\mathrm{DLS}) \\
(\mathbf{n m})^{\mathbf{i}}\end{array}$ & Ref \\
\hline 1 & PNOR & 0.20 & 321 & 1.55 & 17.7 & - & \multirow{16}{*}{$\begin{array}{l}\text { UPy dimeriza- } \\
\text { tion }\end{array}$} & 236 & 14.9 & 15.5 & - & - & 27 \\
\hline 2 & \multirow{5}{*}{ PMMA } & 0.10 & 18.5 & 1.39 & 3.6 & - & & 14.9 & 3.2 & 3.4 & - & - & 28 \\
\hline 3 & & 0.10 & 87.9 & 1.54 & 8.6 & & & 71.8 & 7.6 & 8.0 & & & \\
\hline 4 & & 0.15 & 22.4 & 1.29 & 4.0 & & & 17.4 & 3.5 & 3.6 & & & \\
\hline 5 & & 0.20 & 82.0 & 1.40 & 8.2 & & & 62.0 & 7.0 & 7.2 & & & \\
\hline 6 & & 0.40 & 79.0 & 1.42 & 8.1 & & & 36.0 & 5.2 & 5.9 & & & \\
\hline 7 & \multirow[t]{3}{*}{ PMMA } & 0.10 & 27.9 & 1.12 & 4.5 & 5.0 & & 23.8 & 4.1 & 4.2 & 5.0 & 4.7 & \multirow[t]{7}{*}{30} \\
\hline 8 & & 0.11 & 97.1 & 1.43 & 9.1 & 12.6 & & 87.7 & 8.5 & 8.5 & 11.8 & 11.7 & \\
\hline 9 & & 0.08 & 28.0 & 1.16 & 4.5 & - & & 25.6 & 4.3 & 4.3 & - & - & \\
\hline 10 & PnBA & 0.09 & 89.6 & 1.84 & 8.6 & 9.3 & & 64.5 & 7.2 & 8.1 & 9.9 & 8.8 & \\
\hline 11 & \multirow[t]{2}{*}{ PS } & 0.12 & 27.6 & 1.17 & 4.5 & 4.8 & & 22.5 & 4.0 & 4.2 & 4.4 & 4.4 & \\
\hline 12 & & 0.05 & 33.6 & 1.17 & 5.0 & 6.3 & & 28.4 & 4.5 & 4.8 & 5.7 & 6.1 & \\
\hline 13 & PNOR & 0.07 & 71.8 & 1.26 & 7.6 & 7.3 & & 74.6 & 7.8 & 7.3 & 6.3 & 7.0 & \\
\hline 14 & \multirow[t]{3}{*}{ PIBMA } & 0.04 & 32.6 & 1.16 & 4.9 & \multirow[t]{3}{*}{-} & & 30.7 & 4.7 & 4.8 & \multirow[t]{3}{*}{-} & \multirow[t]{3}{*}{-} & \multirow[t]{3}{*}{32} \\
\hline 15 & & 0.04 & 66.3 & 1.26 & 7.3 & & & 58.4 & 6.8 & 7.1 & & & \\
\hline 16 & & 0.04 & 181.0 & 1.46 & 12.8 & & & 157.3 & 11.9 & 12.5 & & & \\
\hline 17 & \multirow[t]{2}{*}{ POEGMA } & 0.10 & 81.9 & $1.37^{j}$ & - & $7.7^{\mathrm{k}}$ & \multirow{2}{*}{$\begin{array}{l}\text { BTA helical } \\
\text { stacking }\end{array}$} & 77.6 & 1.30 & - & 7.1 & 7.2 & \multirow[t]{2}{*}{22} \\
\hline 18 & & 0.10 & 81.9 & $1.37^{j}$ & - & $7.4^{1}$ & & 77.6 & 1.30 & - & 6.9 & 6.9 & \\
\hline 17 & \multirow[t]{5}{*}{ PMMA } & 0.05 & 55.0 & 1.97 & \multirow[t]{5}{*}{-} & $6.6^{\mathrm{m}}$ & \multirow{5}{*}{$\begin{array}{c}\text { Quadruple } \\
\text { hydrogen } \\
\text { bonding }\end{array}$} & \multirow[t]{5}{*}{-} & - & - & $6.6^{\mathrm{m}}$ & 6.4 & 38 \\
\hline 18 & & 0.10 & 41.6 & 1.67 & & $5.9^{\mathrm{m}}$ & & & & & $5.7^{\mathrm{m}}$ & 5.5 & \\
\hline 19 & & 0.15 & 45.0 & 1.86 & & $5.8^{\mathrm{m}}$ & & & & & $5.1^{\mathrm{m}}$ & 5.3 & \\
\hline 20 & & 0.05 & 112.9 & 2.45 & & $7.9^{\mathrm{m}}$ & & & & & $7.9^{\mathrm{m}}$ & 7.7 & \\
\hline 21 & & 0.10 & 132.0 & 2.87 & & 7 & & & & & $6.6^{\mathrm{m}}$ & 6.6 & \\
\hline 22 & PHEAm & 0.10 & 166 & 1.15 & - & $6.8^{\mathrm{n}}$ & Cucur- & - & - & - & $6.0^{\mathrm{n}}$ & 6.4 & 39 \\
\hline 23 & & 0.10 & 330 & 1.24 & & $10.7^{\mathrm{n}}$ & bit[8]uril & & & & $9.4^{\mathrm{n}}$ & 10.1 & \\
\hline 24 & & 0.10 & 593 & 1.23 & & $12.3^{n}$ & complexation & & & & $10.7^{\mathrm{n}}$ & 11.5 & \\
\hline 25 & & 0.30 & 245 & 1.01 & & $9.2^{\mathrm{n}}$ & & & & & $5.4^{\mathrm{n}}$ & 7.4 & \\
\hline 26 & PHEAm & 0.04 & 71.1 & 1.21 & - & $6.5^{\mathrm{n}}$ & Cyclodextrin- & - & - & - & $5.1^{\mathrm{n}}$ & 6.3 & 40 \\
\hline 27 & & 0.04 & 54.7 & 1.30 & & $5.9^{\mathrm{n}}$ & ferrocene & & & & $4.7^{\mathrm{n}}$ & 5.7 & \\
\hline 28 & & 0.07 & 90.3 & 1.36 & & $6.9^{\mathrm{n}}$ & & & & & $4.4^{\mathrm{n}}$ & 6.6 & \\
\hline 29 & PS & 0.20 & 5.6 & 1.21 & 1.8 & - & Hydrazone & 4.4 & 1.6 & 1.6 & - & - & 41 \\
\hline 30 & & 0.05 & 6.8 & 1.18 & 2.0 & & reversible & 6.7 & 2.0 & 1.9 & & & \\
\hline 31 & & 0.10 & & & 2.0 & & bonds & 6.3 & 1.9 & 1.9 & & & \\
\hline 32 & & 0.20 & & & 2.0 & & & 5.6 & 1.8 & 1.8 & & & \\
\hline 33 & & 0.30 & & & 2.0 & & & 5.9 & 1.9 & 1.6 & & & \\
\hline 34 & & 0.20 & 8.5 & 1.22 & 2.3 & & & 7.4 & 2.1 & 2.0 & & & \\
\hline 35 & & 0.20 & 13.3 & 1.19 & 3.0 & & & 10.2 & 2.6 & 2.6 & & & \\
\hline 36 & & 0.40 & 16.0 & 1.17 & 3.3 & & & 12.4 & 2.8 & 2.4 & & & \\
\hline 37 & & 0.50 & 17.3 & 1.27 & 3.4 & & & 13.9 & 3.0 & 2.3 & & & \\
\hline 38 & PNOR & 0.30 & 50.1 & 1.22 & $4.5^{\circ}$ & - & $\begin{array}{c}\text { Disulfide } \\
\text { reversible } \\
\text { bonds }\end{array}$ & - & $3.9^{\circ}$ & 3.6 & - & - & 42 \\
\hline 39 & PHEMA & 0.10 & 95.4 & 1.24 & - & $4.9^{\mathrm{P}}$ & $\begin{array}{l}\text { Disulfide } \\
\text { exchange }\end{array}$ & - & - & - & $4.3^{\mathrm{P}}$ & 4.6 & 43 \\
\hline 40 & PMMA & 0.30 & 30.9 & 1.05 & - & 3.9 & Enamine & - & - & - & 2.7 & 3.1 & 44 \\
\hline 41 & & 0.30 & 53.5 & 1.05 & & 5.3 & reversible & & & & 3.4 & 4.3 & \\
\hline 42 & & 0.30 & 309.4 & 1.30 & & 13.9 & bonds & & & & 7.1 & 11.2 & \\
\hline
\end{tabular}




\begin{tabular}{|c|c|c|c|c|c|c|c|c|c|c|c|c|c|}
\hline 43 & \multirow[t]{3}{*}{ PMMA } & 0.10 & 30.8 & 1.17 & $4.0^{\circ}$ & \multirow[t]{3}{*}{-} & \multirow{3}{*}{$\begin{array}{c}\text { Anthracene } \\
\text { dimerization }\end{array}$} & \multirow[t]{3}{*}{-} & $3.4^{0}$ & 3.9 & \multirow[t]{3}{*}{-} & \multirow[t]{3}{*}{-} & \multirow[t]{3}{*}{45} \\
\hline 44 & & 0.20 & 30.6 & 1.14 & $3.6^{\circ}$ & & & & $2.9^{\circ}$ & 3.1 & & & \\
\hline 45 & & 0.46 & 42.9 & 1.16 & $3.5^{\circ}$ & & & & $2.3^{\circ}$ & 2.4 & & & \\
\hline 46 & \multirow[t]{4}{*}{ PCOD } & 0.01 & \multirow[t]{4}{*}{33.5} & \multirow[t]{4}{*}{1.34} & \multirow[t]{4}{*}{-} & \multirow[t]{4}{*}{9.3} & \multirow{4}{*}{$\begin{array}{c}\text { Rh- } \\
\text { complexation }\end{array}$} & \multirow[t]{4}{*}{-} & \multirow[t]{4}{*}{-} & \multirow[t]{4}{*}{-} & 8.9 & 9.2 & \multirow[t]{4}{*}{46} \\
\hline 47 & & 0.02 & & & & & & & & & 8.5 & 9.1 & \\
\hline 48 & & 0.05 & & & & & & & & & 8.1 & 9.0 & \\
\hline 49 & & 0.10 & & & & & & & & & 7.4 & 8.6 & \\
\hline 50 & \multirow[t]{4}{*}{ POEGMA } & 0.11 & 110.1 & 1.04 & $7.9^{\circ}$ & \multirow[t]{4}{*}{-} & \multirow{4}{*}{$\begin{array}{c}\mathrm{Cu}- \\
\text { complexation }\end{array}$} & \multirow[t]{4}{*}{-} & $7.0^{\circ}$ & 7.4 & \multirow[t]{4}{*}{ - } & \multirow[t]{4}{*}{-} & \multirow[t]{4}{*}{49} \\
\hline 51 & & 0.20 & 90.4 & 1.10 & $7.1^{\circ}$ & & & & $6.6^{\circ}$ & 6.2 & & & \\
\hline 52 & & 0.35 & 175.0 & 1.08 & $9.8^{\circ}$ & & & & $8.1^{\circ}$ & 7.6 & & & \\
\hline 53 & & 0.40 & 208.0 & 1.05 & $11.0^{\circ}$ & & & & $8.4^{\circ}$ & 8.1 & & & \\
\hline 54 & \multirow[t]{3}{*}{ PS } & 0.03 & \multirow[t]{3}{*}{57.2} & \multirow[t]{3}{*}{1.15} & \multirow[t]{3}{*}{-} & \multirow[t]{3}{*}{6.7} & \multirow{3}{*}{$\begin{array}{l}\text { Cu- } \\
\text { phthalocyani- } \\
\text { ne formation }\end{array}$} & \multirow[t]{3}{*}{-} & - & - & 6.6 & 6.6 & 50 \\
\hline 55 & & 0.09 & & & & & & & & & 5.7 & 6.3 & \\
\hline 56 & & 0.17 & & & & & & & & & 5.3 & 5.9 & \\
\hline 57 & PS & 0.12 & 14.3 & 1.16 & 3.1 & 4.4 & $\begin{array}{c}\text { Pd- } \\
\text { complexation }\end{array}$ & 10.5 & 2.6 & 2.9 & 2.7 & 4.0 & 51 \\
\hline 58 & PCOD & 0.02 & 50.6 & 1.02 & - & 10.1 & Ir- & - & - & - & 9.4 & 9.9 & 52 \\
\hline 59 & & 0.05 & & & & & complexation & & & & 8.6 & 9.8 & \\
\hline 60 & & 0.10 & & & & & & & & & 7.9 & 9.5 & \\
\hline 61 & & 0.10 & 45.3 & 1.03 & - & 10.5 & $\mathrm{Ni}-$ & - & - & - & 9.6 & 9.9 & \\
\hline 62 & & 0.20 & & & & & complexation & & & & 9.1 & 9.2 & \\
\hline 63 & & 0.30 & & & & & & & & & 8.0 & 8.5 & \\
\hline 64 & PHEAm & 0.05 & 43.4 & - & - & $4.5^{n}$ & Fe- & - & - & - & $2.5^{\mathrm{n}}$ & 4.3 & 53 \\
\hline 65 & & 0.10 & 48.9 & & & $4.7^{\mathrm{n}}$ & complexation & & & & $2.4^{\mathrm{n}}$ & 4.4 & \\
\hline
\end{tabular}

${ }^{\text {a }}$ PNOR = Polynorbornene, PMMA = Polymethyl methacrylate, PnBA = Poly( -butyl methacrylate), PS = Polystyrene, PIBMA = Poly(isobornyl methacrylate), POEGMA = Polyoligoethylene glycol methyl ether methacrylate, PHEAm = Poly $(N$-2-hydroxyethyl acrylamide), PHEMA = Poly(hydroxyethyl methacrylate), PCOD = Polycyclooctadiene. ${ }^{\mathrm{b}}$ Relative amount of functional groups in the linear precursor (usually determined by NMR spectroscopy). ${ }^{c}$ Weight average molecular weight referred to PS standards (unless otherwise stated). ${ }^{\mathrm{d}}$ Dispersity of the molecular weight distribution referred to PS standards (unless otherwise stated). ${ }^{\mathrm{e}}$ For the precursors: $R_{H}{ }^{\text {SEC }}(\mathrm{nm})=1.44 \times 10^{-2} M_{w}{ }^{0.561}$ (see ref. 55); for the SCNPs: $R_{H}{ }^{\text {SEC }}$ $(\mathrm{nm})=1.44 \times 10^{-2}\left(M_{w}{ }^{a p p}\right)^{0.561} .{ }^{\mathrm{f}}$ Data from dynamic light scattering (DLS) measurements in THF (unless otherwise stated). ${ }^{\mathrm{g}}$ UPy $=2$-UreidoPyrimidinone. BTA = Benzene-1,3,5-tricarboxamide. ${ }^{\mathrm{h}} M_{w}{ }^{a p p}$ is the apparent weight average molecular weight of the SCNP referred to PS standards (see ref. 56). ${ }^{\mathrm{i}} R_{\mathrm{H}}{ }^{\mathrm{CAL}}(\mathrm{nm})=R_{\mathrm{H}}{ }^{\text {Precursor }}(\mathrm{nm}) \times(1-x)^{0.6}$ (see Eq. 4). ${ }^{\mathrm{j}}$ Data from SEC in DMF $\left(10 \mathrm{mM} \mathrm{LiBr}\right.$ ) referred to PMMA standards. ${ }^{\mathrm{k}}$ Measurements

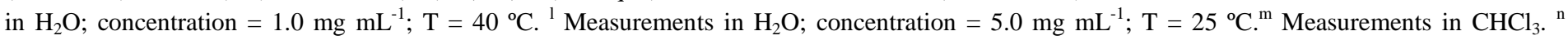
Measurements in $\mathrm{H}_{2} \mathrm{O} .{ }^{\circ}$ Hydrodynamic radius determined by SEC with differential refractive index, multi-angle laser light scattering and viscosimetry detectors. ${ }^{\mathrm{m}}$ Measurements in $\mathrm{MeOH}$. 

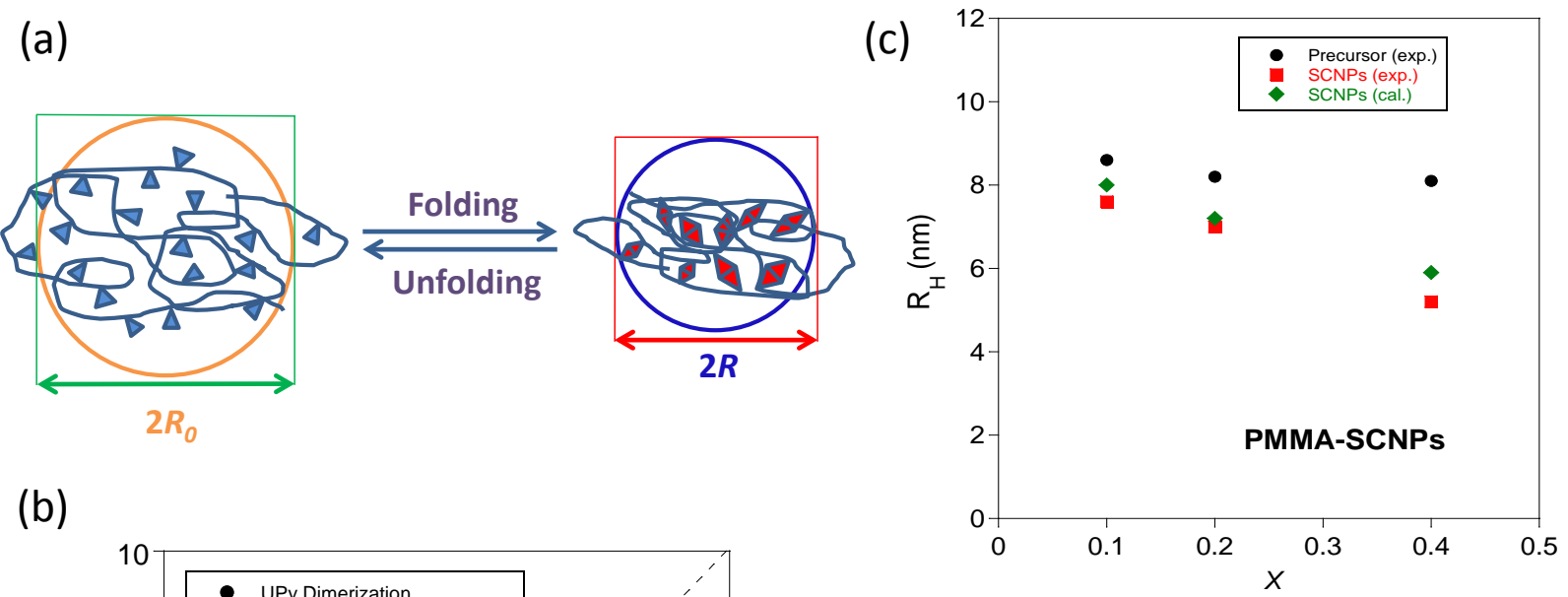

(b)
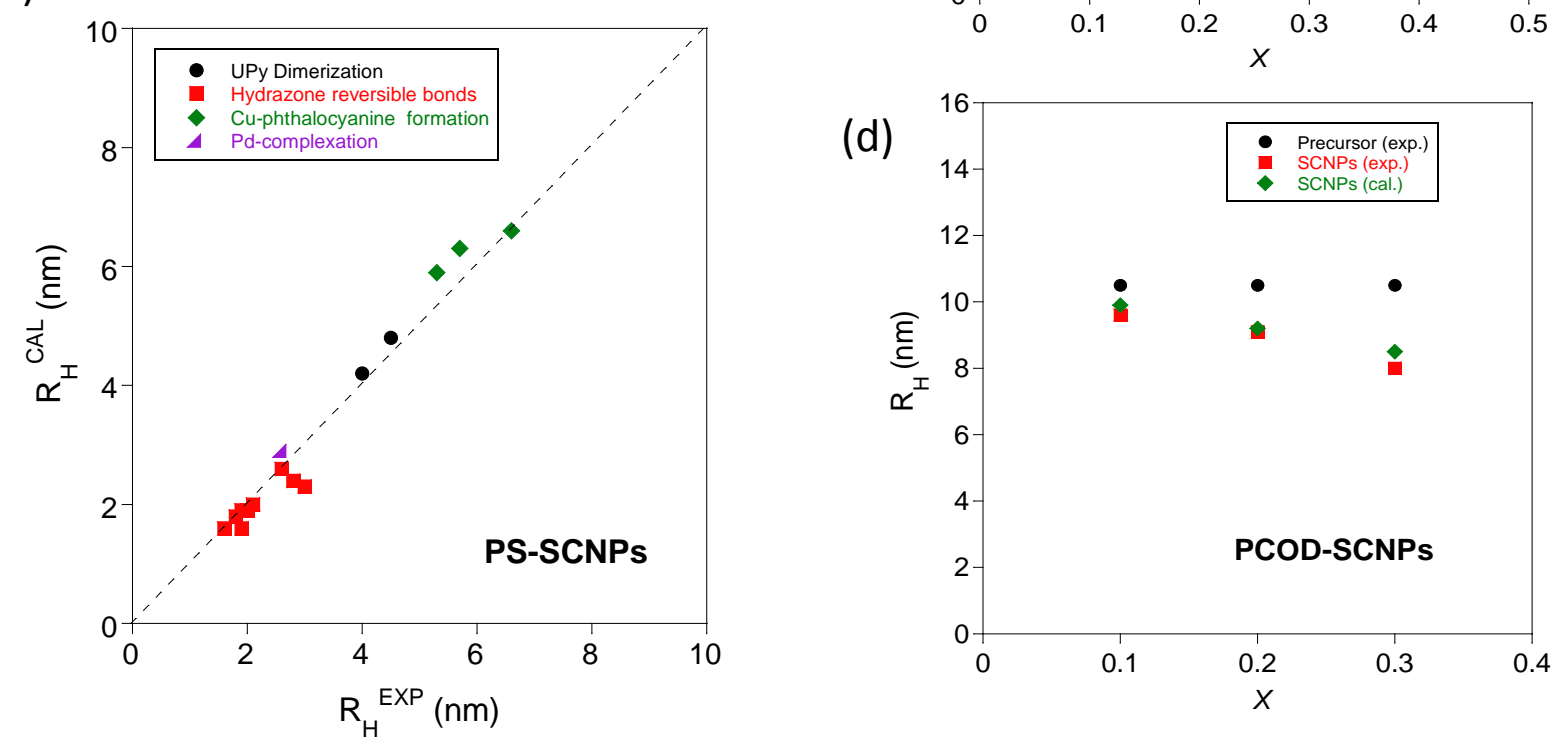

Figure 1. (a) Schematic illustration of the folding of a single chain of size $R_{0}$ to a single-chain nanoparticle of size $R$ via reversible interactions. (b) Comparison of hydrodynamic radius calculated from eq. $4\left(R_{\mathrm{H}}^{\mathrm{CAL}}\right)$ and experimental hydrodynamic radius $\left(R_{\mathrm{H}}^{\mathrm{EXP}}\right)(\mathrm{SEC}$ data from Table 1 unless otherwise stated) for reversible polystyrene (PS)-SCNPs prepared via ureido-pyrimidinone (UPy) dimerization, ${ }^{30}$ hydrazone reversible bonds, ${ }^{41} \mathrm{Cu}$-phthalocyanine formation (DLS data) ${ }^{50}$ and Pd-complexation. ${ }^{51}$ (c) Comparison of $R_{\mathrm{H}}{ }^{\mathrm{CAL}}$ (eq. 4 ) and $R_{\mathrm{H}}{ }^{\text {EXP }}$ for polymethyl methacrylate (PMMA)-SCNPs synthesized via UPy dimerization ${ }^{28}$ as a function of the fraction of groups $x$ involved in reversible bonds. (d) Same as panel (c) but for polycyclooctadiene (PCOD)-SCNPs synthesized via Ni-complexation. ${ }^{52}$

tionally, a comparison of calculated (eq. 4) and experimental hydrodynamic size data for polymethyl methacrylate (PMMA)-SCNPs synthesized via UPy dimerization ${ }^{28}$ and polycyclooctadiene (PCOD)-SCNPs synthesized via Nicomplexation, ${ }^{52}$ as a function of $x$ is illustrated in Figure 1c and $d$, respectively. The general trend of increased size reduction upon increasing $x$ is well captured by eq. 4 .

Figure 2a shows a comparison of hydrodynamic radii calculated from eq. $4\left(R_{\mathrm{H}}{ }^{\mathrm{CAL}}\right)$ and experimental hydrodynamic radii determined by SEC $\left(R_{\mathrm{H}}{ }^{\mathrm{SEC}}\right)$ for reversible PS-, PMMA- , poly(n-butylacrylate) (PnBA)-, poly(isobornyl methacrylate) (PIBMA)-, polynorbornene (PNOR)- and polyoligoethylene glycol methyl ether methacrylate (POEGMA)-SCNPs (data from Table 1). The average deviation of calculated data from experimental ones is $6 \%$. Consequently, based on the hydrodynamic radius of the precursor as determined by SEC, a reliable estimation of the size reduction upon folding the precursor to a SCNP via reversible interactions can be obtained from eq. 4.
However, significant data scatter is observed when experimental hydrodynamic radii from DLS $\left(R_{\mathrm{H}}{ }^{\mathrm{DLS}}\right)$ are compared to hydrodynamic radii calculated from eq. $4\left(R_{\mathrm{H}}{ }^{\mathrm{CAL}}\right)$ (see Figure $2 \mathrm{~b}$ ). It is worth mentioning that the DLS technique is more sensitive to the presence of impurities of large size in solution (e.g., traces of SCNP aggregates) than the SEC technique, ${ }^{54}$ which could account for some of the scatter observed in Figure 2b when compared to Figure 2a. Also, SEC hydrodynamic data are based on the use of highly diluted solutions, whereas DLS hydrodynamic results are often obtained from more concentrated solutions and sometimes involving the use of other solvents different from THF.

Notably, eq. 4 does not reproduce the data of irreversible SCNPs (see Figure 3a, average deviation $\approx 44 \%$ ). This is a consequence of the change in scaling exponent $(v)$ in $R_{\mathrm{H}} \alpha N^{v}$ upon intra-chain covalent-bonded folding/collapse of the precursors to irreversible SCNPs, as reported previously in ref. 54. Hence, a good agreement between theoretical and experimental data is found by assuming a change from $v=0.6$ (pre- 

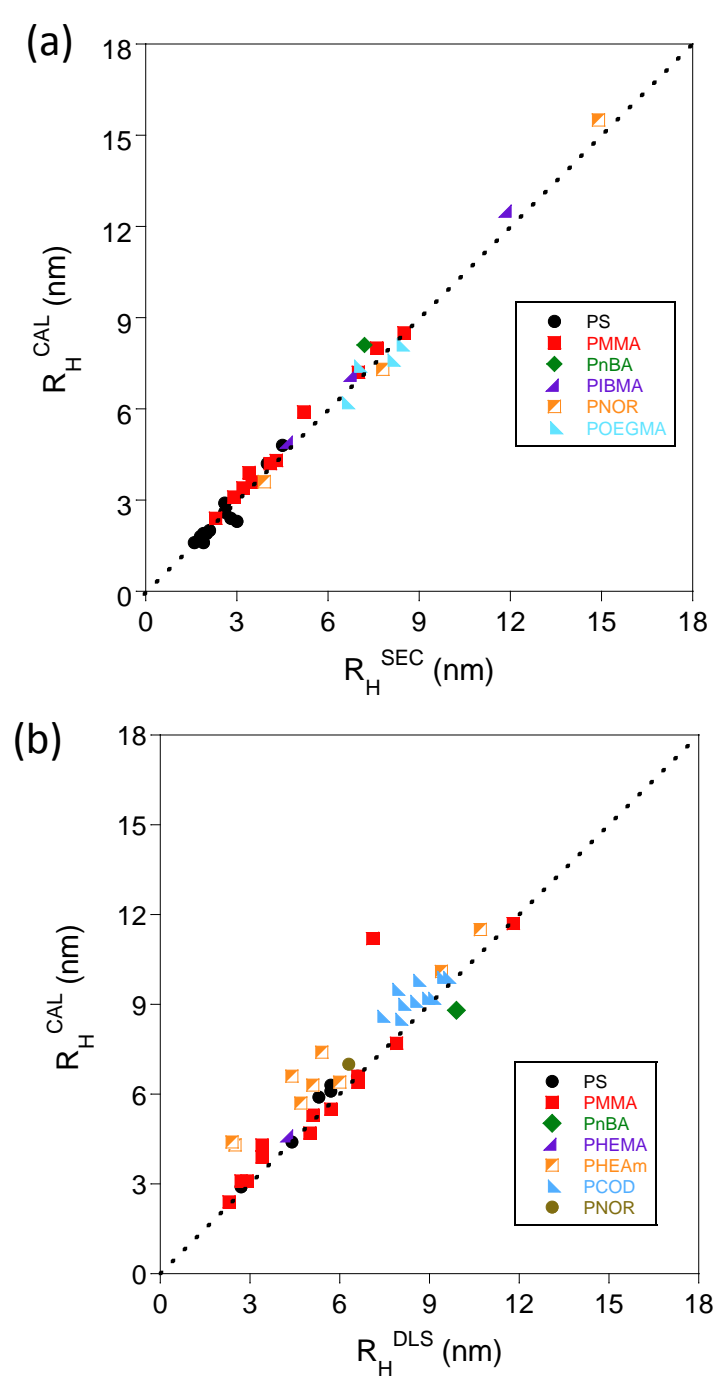

Figure 2. (a) Comparison of $R_{\mathrm{H}}^{\mathrm{CAL}}$ (eq. 4) and experimental $R_{\mathrm{H}}{ }^{\text {SEC }}$ for reversible PS-, PMMA-, PnBA-, PIBMA-, PNOR- and POEGMA-SCNPs. (b) Comparison of $R_{\mathrm{H}}{ }^{\mathrm{CAL}}$ (eq. 4) and experimental $R_{\mathrm{H}}{ }^{\text {DLS }}$ for reversible PS-, PMMA-, PnBA-, PHEMA-, PHEAm- and PNOR-SCNPs.

cursors) to $v=0.53$ (irreversible SCNPs) (see Figure 3b). ${ }^{58}$ The exponent $v=0.53$ is consistent with previous results from experiments and computer simulations of irreversible SCNPs. ${ }^{14,23,47,54,56,59}$

\section{CONCLUSIONS}

In this work, SEC and DLS hydrodynamic size data for different responsive SCNPs, including 65 SCNPs and 16 reversible interactions, have been analyzed. An expression providing the expected size reduction upon folding single chains of size $R_{0}$ to SCNPs of size $R$ via reversible interactions has been derived following a Flory-like argument. The overall agreement between theoretical and experimental data is excellent, hence allowing a valuable a priori estimation of size reduction upon
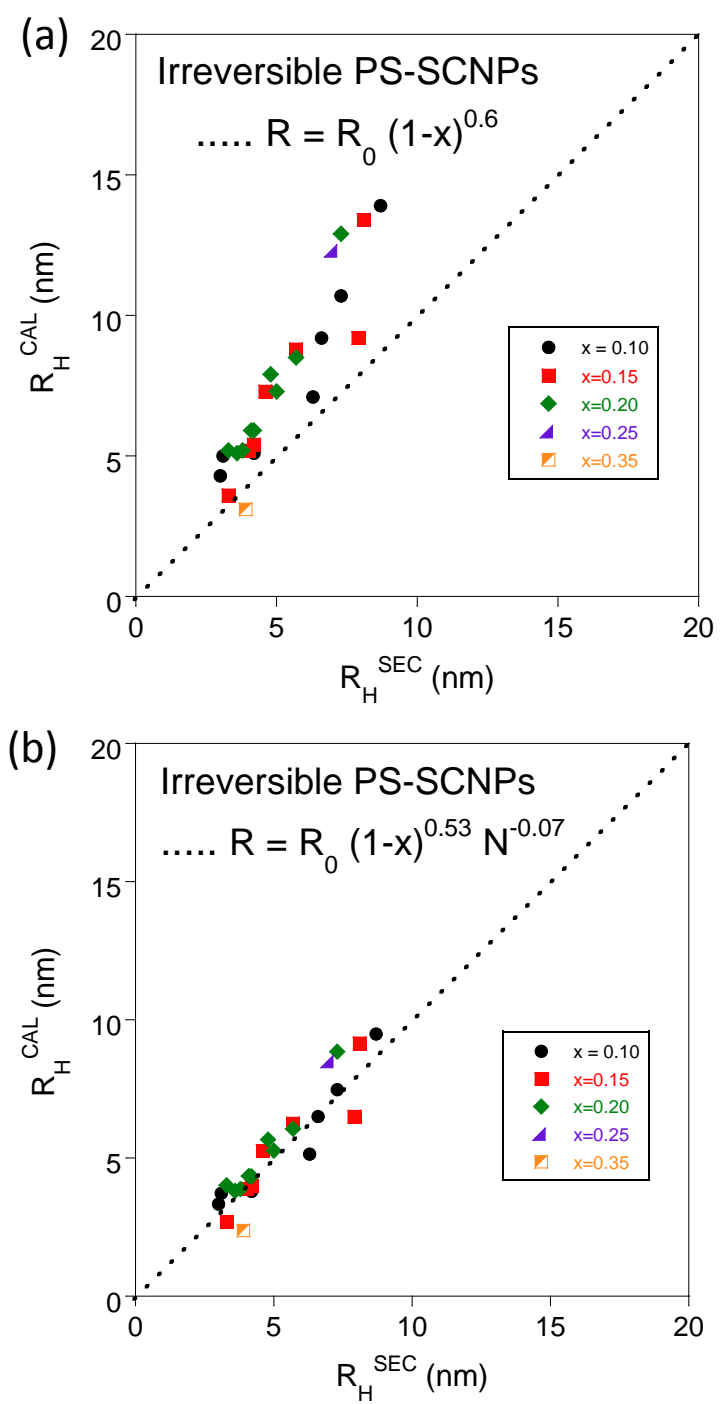

Figure 3. (a) Comparison of $R_{\mathrm{H}}{ }^{\mathrm{CAL}}$ (eq. 4) and experimental $R_{\mathrm{H}}{ }^{\text {SEC }}$ for covalent-bonded (irreversible) PS-SCNPs (data taken from ref. 54). (b) Same as panel (a) but assuming a change in the scaling exponent ( $v$ ) in $R_{\mathrm{H}} \alpha N^{v}$ from $v=0.6$ (precursors) to $v=$ 0.53 (irreversible SCNPs) giving to ${ }^{58} R=R_{0}(1-x)^{0.53} N^{-0.07}$.

folding single chains to single-chain nanoparticles via reversible interactions. When compared to covalent-bonded SCNPs with a non-globular, sparse conformation in solution, reversible SCNPs in solution with similar nature, molar mass and amount of reactive groups display, on average, a lower level of chain compaction. This finding is of outmost importance for developing practical applications of responsive SCNPs based on structure - property relationships.

\section{ASSOCIATED CONTENT}

\section{Supporting Information}

The Supporting Information is available free of charge on the ACS Publications website at DOI: 10.1021/acs.macromol.xxxxxxx. 
Combination of experimental techniques used to validate the singlechain nature of reversible SCNPs reported in Table 1 (PDF)

\section{AUHOR INFORMATION}

\section{Corresponding Author}

*E-mail: josetxo.pomposo@ehu.eus

\section{Notes}

The authors declare no competing financial interest.

\section{ACKNOWLEDGMENTS}

Financial support by the Spanish Ministry "Ministerio de Economia y Competitividad", MAT2015-63704-P (MINECO / FEDER, UE) and the Basque Government, IT-654-13, is acknowledged. J. R.-C. is grateful to the Materials Physics Center MPC for his pre-doctoral grant.

\section{REFERENCES}

(1) Ouchi, M.; Badi, N.; Lutz, J.-F.; Sawamoto, M. Single-chain technology using discrete synthetic macromolecules. Nat. Chem. 2011, 3, 917-924.

(2) Gonzalez-Burgos, M.; Latorre-Sanchez, A.; Pomposo, J. A. Advances in Single Chain Technology. Chem. Soc. Rev. 2015, 44, 6122-6142.

(3) Mavila, S.; Eivgi, O.; Berkovich, I.; Lemcoff, N. G. Intramolecular Cross-Linking Methodologies for the Synthesis of Polymer Nanoparticles. Chem. Rev. 2016, 116, 878-961.

(4) Hanlon, A. M.; Lyon, C. K.; Berda, E. B. What Is Next in SingleChain Nanoparticles? Macromolecules 2016, 49, 2-14.

(5) Altintas, O.; Barner-Kowollik, C. Single-Chain Folding of Synthetic Polymers: A Critical Update. Macromol. Rapid Commun. 2016, 37, 29-46.

(6) Lyon, C. K.; Prasher, A.; Hanlon, A. M.; Tuten, B. T.; Tooley, C. A.; Frank, P. G.; Berda, E. B. A Brief User's Guide to Single-Chain Nanoparticles. Polym. Chem. 2015, 6, 181-197.

(7) Pomposo, J. A. Polymers: Single-Chain Polymer Nanoparticles. In CRC Concise Encyclopedia of Nanotechnology; Kharisov, B. I.; Kharissova, O. V.; Ortiz-Mendez, U., Eds.; CRC Press: Boca Raton, FL, 2015; p 942.

(8) Sanchez-Sanchez, A.; Pomposo, J. A. Single-Chain Polymer Nanoparticles via Non-Covalent and Dynamic Covalent Bonds. Part. Part. Syst. Charact. 2014, 31, 11-23.

(9) Müge, A.; Elisa, H.; Meijer, E. W.; Anja, R. A. P. Dynamic Single Chain Polymeric Nanoparticles: From Structure to Function. In Sequence-Controlled Polymers: Synthesis, Self-Assembly, and Properties; American Chemical Society: 2014; Vol. 1170, pp 313-325.

(10) Sanchez-Sanchez, A.; Perez-Baena, I.; Pomposo, J. A. Advances in Click Chemistry for Single-Chain Nanoparticle Construction. Molecules 2013, 18, 3339-3355.

(11) Altintas, O.; Barner-Kowollik, C. Single Chain Folding of Synthetic Polymers by Covalent and Non-Covalent Interactions: Current Status and Future Perspectives. Macromol. Rapid Commun. 2012, 33, 958-971.

(12) Aiertza, M.; Odriozola, I.; Cabañero, G.; Grande, H.-J.; Loinaz, I. Single-chain polymer nanoparticles. Cell. Mol. Life Sci. 2012, 69, 337-346.

(13) Hamilton, S. K.; Harth, E. Molecular Dendritic Transporter Nanoparticle Vectors Provide Efficient Intracellular Delivery of Peptides. ACS Nano 2009, 3, 402-410.

(14) Sanchez-Sanchez, A.; Akbari, S.; Moreno, A. J.; Lo Verso, F.; Arbe, A.; Colmenero, J.; Pomposo, J. A. Design and Preparation of Single-Chain Nanocarriers Mimicking Disordered Proteins for Combined Delivery of Dermal Bioactive Cargos. Macromol. Rapid Commun. 2013, 34, 1681-1686.

(15) Perez-Baena, I.; Loinaz, I.; Padro, D.; Garcia, I.; Grande, H. J.; Odriozola, I. Single-chain Polyacrylic Nanoparticles with Multiple
Gd(III) Centres as Potential MRI Contrast Agents. J. Mater. Chem. 2010, 20, 6916-6922.

(16) Bai, Y.; Xing, H.; Vincil, G. A.; Lee, J.; Henderson, E. J.; Lu, Y.; Lemcoff, N. G.; Zimmerman, S. C. Practical Synthesis of WaterSoluble Organic Nanoparticles with a Single Reactive Group and a Functional Carrier Scaffold. Chem. Sci. 2014, 5, 2862-2868.

(17) Gillissen, M. A. J.; Voets, I. K.; Meijer, E. W.; Palmans, A. R. A. Single Chain Polymeric Nanoparticles as Compartmentalised Sensors for Metal Ions. Polym. Chem. 2012, 3, 3166-3174.

(18) Latorre-Sanchez, A.; Pomposo, J. A. A simple, fast and highly sensitive colorimetric detection of zein in aqueous ethanol via zeinpyridine-gold interactions. Chem. Commun. 2015, 51, 15736-15738.

(19) Pomposo, J. A. Bioinspired single-chain polymer nanoparticles. Polym. Int. 2014, 63, 589-592.

(20) Huo, M.; Wang, N.; Fang, T.; Sun, M.; Wei, Y.; Yuan, J. Singlechain polymer nanoparticles: Mimic the proteins. Polymer 2015, 66, A11- A21.

(21) Latorre-Sanchez, A.; Pomposo, J. A. Recent bioinspired applications of single-chain nanoparticles. Polym. Int. 2016, 65, 855-860.

(22) Terashima, T.; Mes, T.; De Greef, T. F. A.; Gillissen, M. A. J.; Besenius, P.; Palmans, A. R. A.; Meijer, E. W. Single-Chain Folding of Polymers for Catalytic Systems in Water. J. Am. Chem. Soc. 2011, 133, 4742-4745.

(23) Perez-Baena, I.; Barroso-Bujans, F.; Gasser, U.; Arbe, A.; Moreno, A. J.; Colmenero, J.; Pomposo, J. A. Endowing Single-Chain Polymer Nanoparticles with Enzyme-Mimetic Activity. ACS Macro Lett. 2013, 2, 775-779.

(24) Huerta, E.; Stals, P. J. M.; Meijer, E. W.; Palmans, A. R. A. Consequences of Folding a Water-Soluble Polymer around an Organocatalyst. Angew. Chem., Int. Ed. 2013, 52, 2906-2910.

(25) Tooley, C. A.; Pazicni, S.; Berda, E. B. Toward a Tunable Synthetic [FeFe] Hydrogenase Mimic: Single-Chain Nanoparticles Functionalized with a Single Diiron Cluster. Polym. Chem. 2015, 6, 76467651.

(26) Seo, M.; Beck, B. J.; Paulusse, J. M. J.; Hawker, C. J.; Kim, S. Y. Polymeric Nanoparticles via Noncovalent Cross-Linking of Linear Chains. Macromolecules 2008, 41, 6413-6418.

(27) Foster, E. J.; Berda, E. B.; Meijer, E. W. Metastable Supramolecular Polymer Nanoparticles via Intramolecular Collapse of Single Polymer Chains. J. Am. Chem. Soc. 2009, 131, 6964-6966.

(28) Berda, E. B.; Foster, E. J.; Meijer, E. W. Toward Controlling Folding in Synthetic Polymers: Fabricating and Characterizing Supramolecular Single-Chain Nanoparticles. Macromolecules 2010, 43, 1430-1437.

(29) Foster, E. J.; Berda, E. B.; Meijer, E. W. Tuning the size of supramolecular single-chain polymer nanoparticles. J. Polym. Sci., Part A: Polym. Chem. 2011, 49, 118-126.

(30) Stals, P. J. M.; Gillissen, M. A. J.; Nicolay, R.; Palmans A. R. A.; Meijer, E. W. The balance between intramolecular hydrogen bonding, polymer solubility and rigidity in single-chain polymeric nanoparticles. Polym. Chem. 2013, 4, 2584-2597.

(31) Mes, T.; van der Weegen, R.; Palmans A. R. A.; Meijer, E. W. Single-Chain Polymeric Nanoparticles by Stepwise Folding. Angew. Chem., Int. Ed. 2011, 50, 5085-5089.

(32) Hosono, N.; Gillisen, M. A.; Li, Y.; Sheiko, S. S.; Palmans, A. R. A.; Meijer, E. W. Orthogonal Self-Assembly in Folding Block Copolymers. J. Am. Chem. Soc. 2012, 135, 501-510.

(33) Gillissen, M. A. J.; Terashima, T.; Meijer, E. W.; Palmans, A. R. A.; Voets, I. K. Sticky Supramolecular Grafts Stretch Single Polymer Chains. Macromolecules 2013, 46, 4120-4125.

(34) Hosono, N.; Stals, P. J. M.; Palmans A. R. A.; Meijer, E. W. Consequences of Block Sequence on the Orthogonal Folding of Triblock Copolymers. Chem. Asian J. 2014, 9, 1099-1107.

(35) Stals, P. J. M.; Gillissen, M. A. J.; Paffen, T. F. E.; de Greef, T. F. A.; Lindner, P.; Meijer, E. W.; Palmans, A. R. A.; Voets, I. K. Folding Polymers with Pendant Hydrogen Bonding Motifs in Water: The Effect of Polymer Length and Concentration on the Shape and 
Size of Single-Chain Polymeric Nanoparticles. Macromolecules 2014, 47, 2947-2954.

(36) Hosono, N.; Palmans A. R. A.; Meijer, E. W. "Soldier-SergeantSoldier" triblock copolymers: revealing the folded structure of singlechain polymeric nanoparticles. Chem. Commun. 2014, 50, 7990-7993. (37) Huerta, E.; van Genabeek, B.; Stals, P. J. M.; Meijer, E. W.; Palmans, A. R. A. A modular approach to introduce function into single-chain polymeric nanoparticles. Macromol. Rapid Commun. 2014, 35, 1320-1325.

(38) Wang, F.; Pu, H.; Jin, M.; Pan, H.; Chang, Z.; Wan, D.; Du, J. From single-chain folding to polymer nanoparticles via intramolecular quadruple hydrogen-bonding interaction. J. Polym. Sci., Part A: Polym. Chem. 2015, 53, 1832-1840.

(39) Appel, E. A.; Dyson, J.; del Barrio, J.; Walsh, Z.; Scherman, O. A. Formation of Single-Chain Polymer Nanoparticles in Water through Host-Guest Interactions. Angew. Chem., Int. Ed. 2012, 51, 4185-4189.

(40)Wang, F.; Pu, H.; Che, X. Voltage-responsive single-chain polymer nanoparticles via host-guest interaction. Chem. Commun. 2016, 52, 3516-3519.

(41) Murray, B. S.; Fulton, D. A. Dynamic Covalent Single-Chain Polymer Nanoparticles. Macromolecules 2011, 44, 7242-7252.

(42) Tuten, B. T.; Chao, D.; Lyon, C. K.; Berda, E. B. Single-chain polymer nanoparticles via reversible disulfide bridges. Polym. Chem. 2012, 3, 3068-3071.

(43) Song, C.; Li, L.; Dai, L.; Thayumanavan, S. Responsive singlechain polymer nanoparticles with host-guest features. Polym. Chem. 2015, 6, 4828-4834.

(44) Sanchez-Sanchez, A.; Fulton, D. A.; Pomposo, J. A. pHresponsive single-chain polymer nanoparticles utilising dynamic covalent enamine bonds. Chem. Commun. 2014, 50, 1871-1874.

(45) Frank, P. G.; Tuten, B. T.; Prasher, A.; Chao, D.; Berda, E. B. Intra-Chain Photodimerization of Pendant Anthracene Units as an Efficient Route to Single-Chain Nanoparticle Fabrication. Macromol. Rapid Commun. 2014, 35, 249-253.

(46) Mavila, S.; Diesendruck, C. E.; Linde, S.; Amir, S.; Shikler, R.; Lemcoff, N. G. Polycyclooctadiene Complexes of Rhodium(I): Direct Access to Organometallic Nanoparticles. Angew. Chem. Int. Ed. 2013, 52, 5767-5770.

(47) Sanchez-Sanchez, A.; Arbe, A.; Colmenero, J.; Pomposo, J. A. Metallo-Folded Single-Chain Nanoparticles with Catalytic Selectivity. ACS Macro Lett. 2014, 3, 439-443.

(48) Sanchez-Sanchez, A.; Arbe, A.; Kohlbrecher, J.; Colmenero, J.; Pomposo, J. A. Efficient Synthesis of Single-Chain Globules Mimicking the Morphology and Polymerase Activity of Metalloenzymes. Macromol. Rapid Commun. 2015, 36, 1592-1597.

(49) Basasoro, S.; Gonzalez-Burgos, M.; Moreno, A. J.; Lo Verso, F.; Arbe, A.; Colmenero, J.; Pomposo, J. A. A Solvent-Based Strategy for Tuning the Internal Structure of Metallo-Folded Single-Chain Nanoparticles. Macromol. Rapid Commun. 2016, 37, 1060-1065.

(50) Jeong, J.; Lee, Y.-J.; Kim, B.; Kim, B.; Jung, K.-S.; Paik, H.-J. Colored single-chain polymeric nanoparticles via intramolecular copper phthalocyanine formation. Polym. Chem. 2015, 6, 3392-3397.

(51) Willenbacher, J.; Altintas, O.; Trouillet, V.; Knöfel, N.; Monteiro, M. J.; Roesky, P. W.; Barner-Kowollik, C. Pd-complex driven formation of single-chain nanoparticles. Polym. Chem. 2015, 6, 43584365.

(52) Mavila, S.; Rozenberg, I.; Lemcoff, N. G. A general approach to mono- and bimetallic organometallic nanoparticles. Chem. Sci. 2014, 5, 4196-4203

(53) Wang, F.; Pu, H.; Jin, M.; Wan, D. Supramolecular Nanoparticles via Single-Chain Folding Driven by Ferrous Ions. Macromol. Rapid Commun. 2016, 37, 330-336.

(54) Pomposo, J. A.; Perez-Baena, I.; Lo Verso, F.; Moreno, A. J.; Arbe, A.; Colmenero, J. How Far Are Single-Chain Polymer Nanoparticles in Solution from the Globular State? ACS Macro Lett. 2014, $3,767-772$.
(55) Fetters, L. J.; Hadjichristidis, N.; Lindner, J. S.; Mays, J. W. Molecular Weight Dependence of Hydrodynamic and Thermodynamic Properties for Well-Defined Linear Polymers in Solution. J. Phys. Chem. Ref. Data 1994, 23, 619-640.

(56) Pomposo, J. A.; Perez-Baena, I.; Buruaga, L.; Alegría, A.; Moreno, A. J.; Colmenero, J. On the Apparent SEC Molecular Weight and Polydispersity Reduction upon Intramolecular Collapse of Polydisperse Chains to Unimolecular Nanoparticles. Macromolecules 2011, 44, 8644-8649.

(57) Rubinstein, M.; Colby, R. H. Polymer Physics; Oxford University Press, Inc.: New York, 2003.

(58) Let us consider the case of irreversible SCNP formation in which a change in scaling exponent takes place from $v_{0}$ (precursor) to $v$ (SCNPs). Then, eq. 4 becomes:

$$
\frac{R}{R_{0}}=k(1-x)^{v} N^{\left(v-v_{0}\right)} \approx(1-x)^{v} N^{\left(v-v_{0}\right)} \text { by assuming } k \approx 1 .
$$

(59) Moreno, A. J.; Lo Verso, F.; Sanchez-Sanchez, A.; Arbe, A.; Colmenero, J.; Pomposo, J. A. Advantages of Orthogonal Folding of Single Polymer Chains to Soft Nanoparticles. Macromolecules 2013, 46, 9748-9759. 